\title{
Triple-Point Fermions in Ferroelectric GeTe
}

\author{
Juraj Krempaský $\odot,{ }^{1, \dagger}$ Laurent Nicolaï $\odot,{ }^{2}$ Martin Gmitra $\odot,{ }^{3, *}$ Houke Chen $\odot,{ }^{4}$ Mauro Fanciulliø ${ }^{5}$ \\ Eduardo B. Guedes $\odot,{ }^{1}$ Marco Caputo $\odot,{ }^{1}$ Milan Radović, ${ }^{1}$ Valentine V. Volobuev $\odot,{ }^{6,7}$ Ondřej Caha, ${ }^{8}$ \\ Gunther Springholz®, ${ }^{9}$ Jan Minár, ${ }^{2}$ and J. Hugo Dil $\oplus^{1,10}$ \\ ${ }^{1}$ Photon Science Division, Paul Scherrer Institut, CH-5232 Villigen, Switzerland \\ ${ }^{2}$ New Technologies-Research Center, University of West Bohemia, 30100 Plzeň 3, Czech Republic \\ ${ }^{3}$ Institute of Physics, P. J. Šafárik University in Košice, Park Angelinum 9, 04001 Košice, Slovakia \\ ${ }^{4}$ Department of Physics, Tsinghua University, Beijing 100084, China \\ ${ }^{5}$ Laboratoire de Physique des Matériaux et Surfaces, CY Cergy Paris Université, 95031 Cergy-Pontoise, France \\ ${ }^{6}$ International Research Centre MagTop, Institute of Physics, Polish Academy of Sciences, \\ Aleja Lotnikow 32/46, PL-02668 Warsaw, Poland \\ ${ }^{7}$ National Technical University "KhPI", Kyrpychova Street 2, 61002 Kharkiv, Ukraine \\ ${ }^{8}$ Department of Condensed Matter Physics, Masaryk University, Kotlárská 267/2, 61137 Brno, Czech Republic \\ ${ }^{9}$ Institut für Halbleiter-und Festkörperphysik, Johannes Kepler Universität, A-4040 Linz, Austria \\ ${ }^{10}$ Institut de Physique, École Polytechnique Fédérale de Lausanne, CH-1015 Lausanne, Switzerland
}

(Received 13 November 2020; revised 16 February 2021; accepted 8 April 2021; published 17 May 2021)

Ferroelectric $\alpha$-GeTe is unveiled to exhibit an intriguing multiple nontrivial topology of the electronic band structure due to the existence of triple-point and type-II Weyl fermions, which goes well beyond the giant Rashba spin splitting controlled by external fields as previously reported. Using spin- and angleresolved photoemission spectroscopy combined with ab initio density functional theory, the unique spin texture around the triple point caused by the crossing of one spin-degenerate and two spin-split bands along the ferroelectric crystal axis is derived. This consistently reveals spin winding numbers that are coupled with time-reversal symmetry and Lorentz invariance, which are found to be equal for both triple-point pairs in the Brillouin zone. The rich manifold of effects opens up promising perspectives for studying nontrivial phenomena and multicomponent fermions in condensed matter systems.

DOI: 10.1103/PhysRevLett.126.206403

The exploration of topological materials commenced with the discovery of topological insulators [1-10], followed by topological semimetals [11-14], topological superconductors [15], and nodal line semimetals [16]. Recently, topological semimetals with triply degenerate points were predicted in materials such as molybdenum phosphide [17], tungsten carbide [18], InAs ${ }_{1-x} \mathrm{Sb}_{x}$ alloys [19], and strained HgTe [20]. In contrast to an even number of band crossings, band structures with triple band crossing are rather rare in condensed matter. Such triple-point (TP) fermions can be found along the high-symmetry axis in the Brillouin zone (BZ) allowing for two- and one-dimensional double group representations $[19,21,22]$. This is also the case for $\alpha$-GeTe(111), which was so far extensively studied in terms of the topologically trivial Rashba-type band structure [23-25].

The origin of these intriguing features in $\alpha$-GeTe lies in the spontaneous structural phase transition of the inversion symmetric rocksalt $\beta$-GeTe structure (fcc, space group 225) into a ferroelectric rhombohedral structure with $\mathrm{Ge}$ atoms displaced by $\approx 0.3 \AA$ and distorted by $\approx 1.6^{\circ}$ (space group $160)[24,26]$. This inversion symmetry breaking introduces a spin splitting except for the bands that line up with the high-symmetry ferroelectric $\langle 111\rangle$ axis, which is the main symmetry axis of the $C_{3 v}$ point group. Along this axis, TP fermions can form which are classified as type $A$ or $B$, depending on the number of nodal lines that connect a pair of TPs.

In this Letter, we provide theoretical and experimental evidence that $\alpha$-GeTe possess type- $B$ triple-point fermions inside its valence band states along the ferroelectric $\langle 111\rangle$ axis. To date, such nontrivial topology in $\alpha$-GeTe failed to be noticed. The spin texture around the TP is unraveled, and the same spin winding number of \pm 1 is found for both TP pairs in the 3D BZ. Furthermore, we identified additional Dirac and type-II Weyl fermions that coexist with these three-component fermions.

Epitaxial films of $200 \mathrm{~nm}$ of $\alpha$-GeTe for our (spin-) and angle-resolved photoemission spectroscopy [(S)ARPES] studies were grown by molecular beam epitaxy on $\mathrm{BaF}_{2}(111)$ substrates SARPES measurements were performed at the Swiss Light Source of the Paul Scherrer Institut at the COmplete PHotoEmission Experiment (COPHEE) end station equipped with two orthogonal Mott polarimeters to measure the three spatial components of spinors in arbitrary reciprocal space points [27]. 
Complementary high-resolution ARPES was performed at the UE112-PGM2a beam line of BESSY II. We compared our experimental data with calculated initial state band maps presented in terms of Bloch spectral functions (BSFs) as implemented in the fully relativistic spin-polarized Korringa-Kohn-Rostoker theory [28,29] and with the full potential all-electron Wien $2 \mathrm{~K}$ density functional code. Considering the relatively small energy scales involved, this allowed us to check the results for consistency.

Figure 1(a) shows the BZ with two pairs of triple points, $\mathrm{TP}_{z}^{\sigma}$, where the $\sigma= \pm$ index denotes the TP within a single pair and $z= \pm$ denotes the two TP pairs with opposite $k_{z}$ within the BZ. Figure 1(b) shows the calculated valence band (VB) dispersions for 16 selected $k_{z}$ values in the $Z-A-\Gamma$ plane. The top-left image presents the Rashba-type spin splitting of the uppermost VB at the $Z$ point. When moving $k_{z}$ toward $\Gamma$, these bands disperse and finally meet with the lower spin-split bands at approximately $\frac{1}{3} \mathrm{BZ}$ distance from the $\Gamma$ point (orange arrow). Another visualization of the TP formation is in Fig. 1(c) plotting the dispersion along $\Gamma Z$. At first glance, this crossing point is reminiscent of the topologically protected touching point between electron and hole pockets in a double-Weyl scenario [30-32], but Figs. 2(b)-2(e) unambiguously resolve a three-component fermion matching the space group symmetry. Besides this, the bulk electronic structure in the $U^{\prime}-Z-U$ mirror plane for
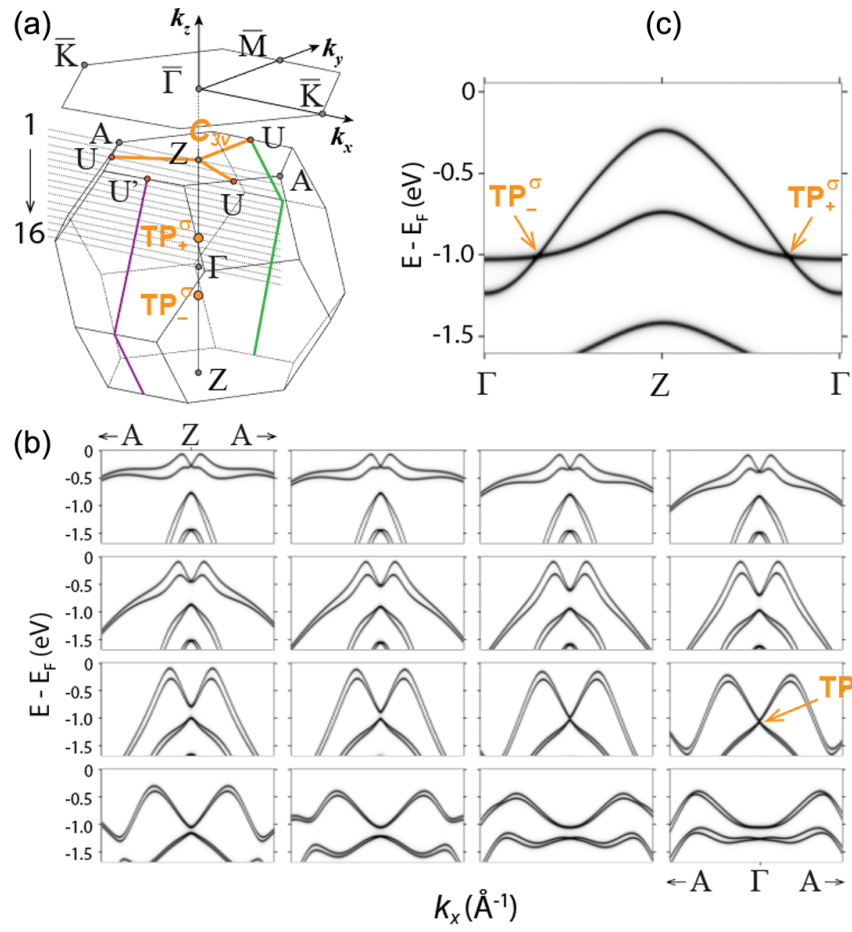

FIG. 1. (a) Bulk BZ and surface BZ of rhombohedral $\alpha$-GeTe with $\langle 111\rangle$ ferroelectric axis along the $k_{z}$ direction. (b) BSF initial state calculations along $k_{x}$ for 16 selected $k_{z}$ values denoted in (a). (c) Band structure along $k_{z}$ with the position of $\mathrm{TP}_{-}^{\sigma}$ and $\mathrm{TP}_{+}^{\sigma}$ indicated by orange arrows. a $k_{z}$ right at the TP in Fig. 2(a) shows two additional band crossings with nontrivial topology. First, there is a Dirac point (DP) near $2 \mathrm{eV}$ binding energy. Second, a type-II Weyl point (WP II) appears around $-0.4 \AA^{-1}$ as indicated in the figure. The presence of this Weyl point influences the spin texture and changes the spin winding number from \pm 1 to \pm 2 , as seen in Figs. 5(e) and 5(f) and discussed in more detail below.

Following the TP classification by Winkler, Singh, and Soluyanov [21], $\alpha$-GeTe should host a type- $B$ TP with four nodal lines. Figure 2 summarizes this scenario: Fig. 2(b) resolves the bands $\Lambda_{4,5,6}$ dispersing in $k_{z}$, whereby the two spin-split bands $\Lambda_{4}$ and $\Lambda_{5}$ cross the doubly degenerate $\Lambda_{6}$, forming a pair of triple-fermion quasiparticles. In contrast to type- $A$ TPs with only one nodal line, the triple points within each pair $\mathrm{TP}_{-}^{\sigma}$ and $\mathrm{TP}_{+}^{\sigma}$ are connected by four nodal lines. One nodal line is lined up with the $C_{3 v}$ axis and, therefore, shows up at zero in-plane momentum only
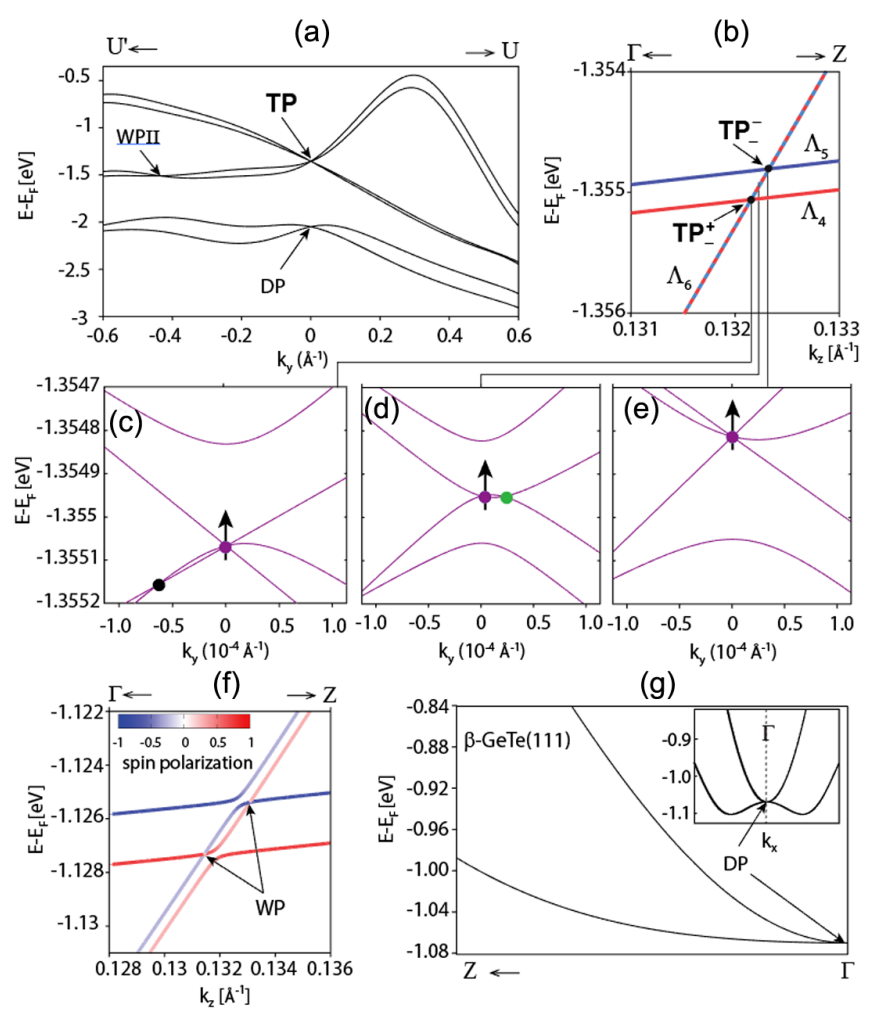

(g)

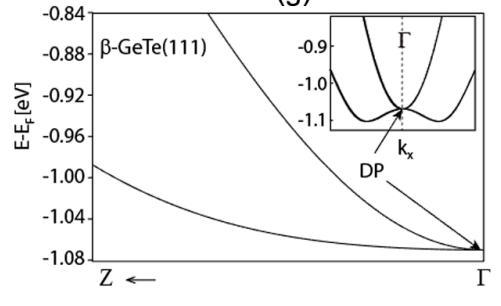

FIG. 2. (a) Calculated band structure along the $U^{\prime}-Z-U$ mirror plane, with TP, WP II, and DP. (b) Formation of a TP pair $\left(\mathrm{TP}_{-}^{-}, \mathrm{TP}_{-}^{+}\right)$by spin-degenerate band $\Lambda_{6}$ and two spin-split bands $\Lambda_{4,5}$. (c)-(e) Close-up of band structure along $k_{y}$ at three different values of $k_{z}$ across the TP pair. Three types of touching points are indicated with black, green, and magenta markers, and black arrows indicate the $k_{z}$ dispersion of the latter. (f) Band structure around the TP under $10 \mathrm{~T}$ magnetic field along the $z$ direction. The TP splits into a pair of Weyl points (WPs). (g) Band structure for rocksalt $\beta$-GeTe above the ferroelectric phase transition. The triple fermions merges into a single DP. Inset: band structure along $k_{x}$. 
[purple markers in Figs. 2(c)-2(e)]. On the other hand, the three remaining nodal lines are extending in three vertical mirror planes, which in our case line up along the three $Z-U$ directions [orange lines in Fig. 1(a)]. Figures 2(c)-2(e) are close-ups of the band structure across the single $\mathrm{TP}_{-}^{ \pm}$ pair for three $k_{z}$ values. For the intermediate $k_{z}$ between $\mathrm{TP}_{-}^{-}$and $\mathrm{TP}_{-}^{+}$, there are two crossing points forming two of the four nodal lines, whereby the one at finite $k_{\|}$(green marker) disperses with $k_{z}$ (see animated Fig. S1 in Supplemental Material [33]).

As a whole, the triple fermion forms an intermediate state between Dirac and Weyl fermions. Actually, as indicated in Fig. 2(f), for $\alpha$-GeTe inside a $10 \mathrm{~T}$ magnetic field [which is many orders of magnitude lower than the exchange field in (GeMn)Te [34]], the TP splits into a pair of Weyl points. Furthermore, above the ferroelectric transition temperature $\left(T_{C} \approx 700 \mathrm{~K}\right)$, the TP transforms into a Dirac point as seen in Fig. 2(g). This renders GeTe rather unique, as all three phases can be achieved under realistic conditions in a single material system.

In our earlier $\alpha$-GeTe ARPES studies, we extensively described the measured electronic structure in terms of surface states (SSs), surface resonances (SRs), and bulk states (B) [24,24,25,34-36]. Such a decomposition also applies to the ARPES data in Fig. 3 and allows one to find the appropriate region for measuring the TP and its spin texture. Below, we demonstrate that inside the $-0.2<k_{y}<$ $0.2 \AA^{-1}$ momentum span the additional spin tag in SARPES data enables us to unambiguously resolve the individual bulk bands around a TP pair.

Based on the $k_{z}$ series of band dispersions in Fig. 1(b), the ARPES data in Fig. 3 , and the schematic $k_{z}$ vs photon energy diagram (Supplemental Fig. S2 [33]), the TP is predicted, and experimentally verified, for photon energies around $h \nu=30,58$, and $89 \mathrm{eV}$. The latter two values

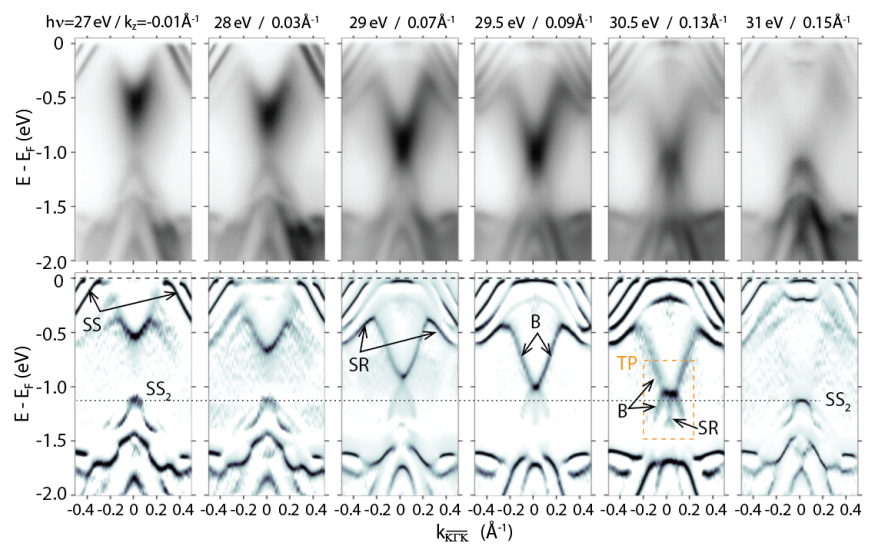

FIG. 3. Top: high-resolution ARPES data for various photon energies around the TP (with corresponding reduced $k_{z}$ values with respect to $\Gamma$ ). Bottom: corresponding curvature maps showing the TP formation at $30.5 \mathrm{eV}$ photon energy inside the orange frame. The arrows indicate surface states $\mathrm{SS}_{2}, \mathrm{SR}$, and $\mathrm{B}$. correspond to consecutive TP pairs at opposite parts of the $\mathrm{BZ}$, thus enabling us to experimentally examine their symmetry connection.

A notable feature of the TP electronic structure is that the bands gradually shift between $\mathrm{TP}_{-}^{-}$and $\mathrm{TP}_{-}^{+}$as marked by black arrows in Figs. 2(c)-2(e). This spectral weight transfer across the TP is manifested in a series of high-resolution ARPES band maps in Fig. 3 and their curvature plots [37] in the bottom panels. In accord with the theoretical predictions [Fig. 1(b)], the $k_{z}$ dispersion of the spectral weight is progressing towards the TP around $30.5 \mathrm{eV}$, where it becomes equally distributed above and below the TP. Interestingly, the band maps inside the orange frame for $h \nu=30.5 \mathrm{eV}$ appear to mimic the TP band mixing seen in Figs. 2(c)-2(e), however with much larger momentum and energy scale. Because the bulk spectral intensity around the TP totally suppresses the weakly contributing surface states $\mathrm{SS}_{2}$ (horizontal dashed line in Fig. 3), the contribution of the latter can be neglected in our SARPES analysis. We note that surface states are typically associated with nodal points in topological metals and semimetals, but $\mathrm{SS}_{2}$ is not interconnecting the TP pairs, because it is clearly offset in energy at zero momentum, as indicated inside the orange frame. In fact, because both TP pairs line up in the $\Gamma Z$ direction, the associated surface states project on the same in-plane momentum and, thus, cannot show up in ARPES experiments on the (111) surface.

Similar to the Rashba model, the spin polarization of bulk states around a TP is expected to be tangentially momentum-locked in accord with time-reversal symmetry (TRS) properties, as also shown in the calculated spin texture in Fig. 5(e). Our SARPES data are visualized as spin-resolved energy distribution (EDC) and momentum distribution curves (MDC) summarized in Figs. 4 and 5, respectively. For all measurements, our analysis concentrates on the in-plane spin polarization $P_{x, y}$ of the four spinsplit bulk bands denoted by $B_{1,2,3,4}$. Their properties are determined using a $3 \mathrm{D}$ vectorial fit of the measured spin expectation values with a well-established fitting routine that extracts their positions, widths, intensities, and 3D spin vector [38]. For the EDCs a Shirley background is subtracted from the total intensity, whereas for the MDCs a constant background is used. To simplify comparison between theory and experiment, we aligned the sample in such a way that the out-of-plane spin polarization $P_{z}$ is oriented along the $k_{z}$ direction and $P_{x}\left(P_{y}\right)$ along the $\overline{\Gamma K}(\overline{\Gamma M})$ direction. Within this experimental geometry, a tangential spin winding is determined by $P_{x}$. Under the experimental conditions required for measuring the TP, it is not possible to extract the degree of initial state spin polarization [36,39-42], and we thus focus on the spin texture.

We first test the spin texture in terms of TRS and Lorentz invariance (LI) for the selected $k_{x, y}$ point labeled $A$ in 


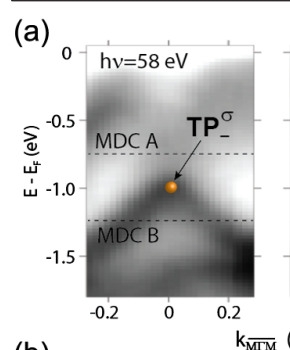

(b)

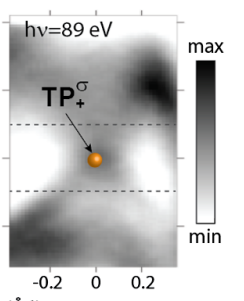

(c)
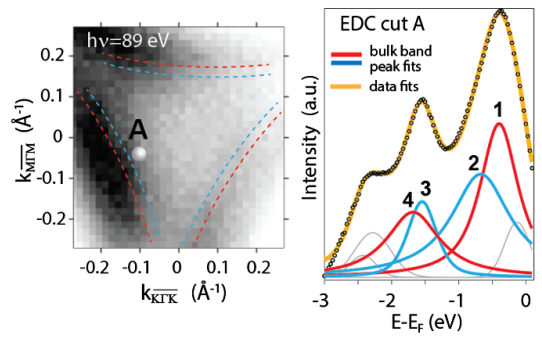
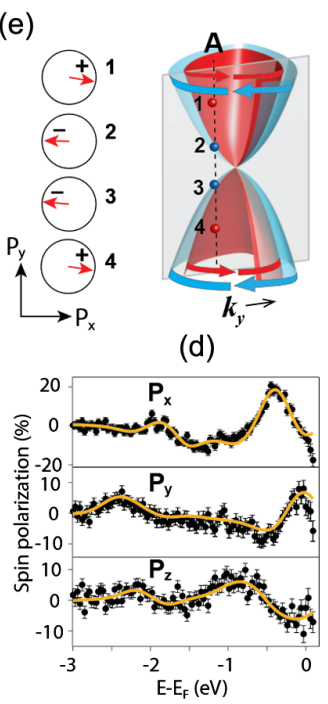

FIG. 4. (a) ARPES band maps along $\overline{M \Gamma M}$ measured at the COPHEE setup with $h \nu=58$ and $89 \mathrm{eV}$. Orange bullets indicate $\mathrm{TP}_{-}^{\sigma}$ and $\mathrm{TP}_{+}^{\sigma}$. MDC $A$ and MDC $B$ indicate where data in Fig. 5 are obtained. (b) Constant energy map (CEM) measured at $h \nu=$ 89 and $-0.4 \mathrm{eV}$ binding energy at COPHEE. Marker $A$ indicates the $k_{x, y}$ locus used for SARPES EDC, next to the bulk bands denoted with dashed red and blue lines. (c),(d) SARPES EDC cut $A$ total intensity and related $P_{x, y, z}$ spin polarizations with corresponding $B_{1,2,3,4}$ bulk band fitting indicated in red and blue and surface-derived bands in gray. (e) 3D TP cartoon illustrating EDC cut $A$ with corresponding in-plane spin textures, showing spin vectors projected mainly along $P_{x}$ with a characteristic $(+--+)$ pattern around the TP.

Fig. 4(b), with SARPES data summarized in Figs. 4(c) and 4(d). The resulting spin texture is summarized in Fig. 4(e) next to a model visualizing the EDC cut through the four

$B_{1-4}$ bulk bands around the TP. We note that the spin helicity dictated by TRS and LI are preserved: The spins are nearly tangentially locked in momentum, whereby bands $B_{1,4}$ and $B_{2,3}$ do not change the spin helicity. The spin texture imposed by LI, i.e., the same spin and momentum when the energy is inverted with regard to the TP, becomes apparent when considering the $(+--+)$ pattern in Fig. 4(d); in contrast to the Rashba-type $(+-+-)$ pattern seen in Supplemental Fig. S3 [33], measured at the BZ boundary $Z$ point [see Fig. 1(a)].

This experimental result is further confirmed in Fig. 5. We measured each TP pair with two MDC cuts $250 \mathrm{meV}$ above $(A)$ and below $(B)$ the TP, as depicted in Fig. 4(a). In addition, because there are two inequivalent directions [Fig. 1(a), green and magenta lines], each TP pair was measured along $U^{\prime}-A-U$ and after rotation by $180^{\circ}$ along $U-A-U^{\prime}$ (see SARPES data in Supplemental Fig. S4 [33]). The resulting in-plane spin textures shown in Fig. 5(d) are consistent with a spin winding number of \pm 1 . To the best of our knowledge, this is the first experimental evidence of the spin texture of a triple fermion, featuring a winding number of \pm 1 in accord with both TRS and LI and theoretical predictions. Here, it should be noted that the inclusion of WP II induces an additional spin swirl and changes the winding number to \pm 2 as illustrated by the calculations in Fig. 5(e). Furthermore, the two triple-point pairs $\mathrm{TP}_{-}^{\sigma}$ and $\mathrm{TP}_{+}^{\sigma}$ show the same spin helicity in accordance with the absence of an $(x, y)$ mirror plane.

In conclusion, we demonstrated that $\alpha$-GeTe is a type- $B$ triple-fermion topological system enabled by the ferroelectric noncentrosymmetric atomic arrangement of $\mathrm{Ge}$ and $\mathrm{Te}$ atoms along the $\langle 111\rangle$ axis. Furthermore, we measured for the first time the spin winding number for a triple-point (a)

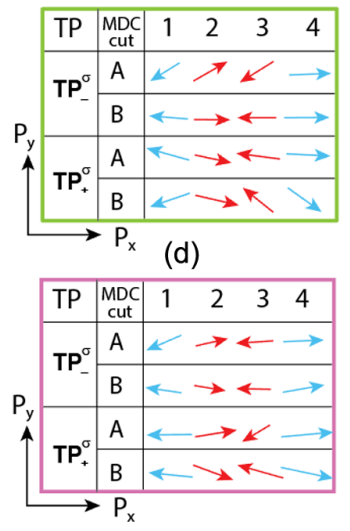

(b)

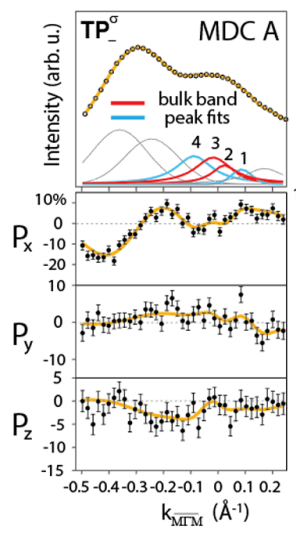

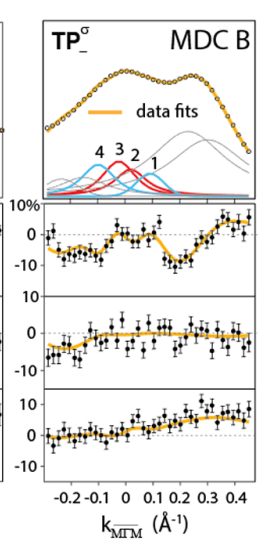

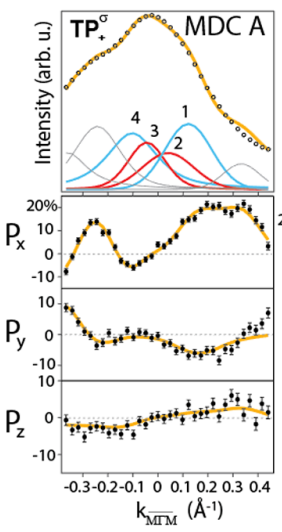

(c)

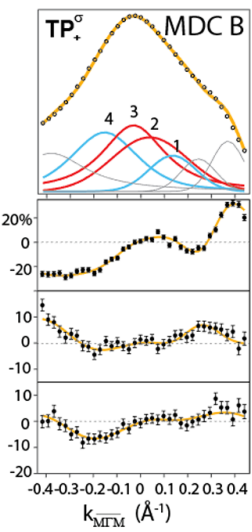

(e)

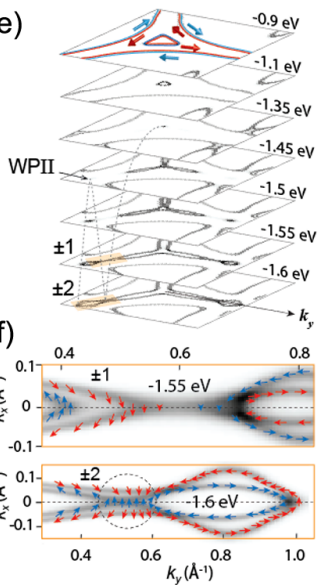

FIG. 5. (a) Tabulated spin textures for $\mathrm{TP}_{-}^{\sigma}$ and $\mathrm{TP}_{+}^{\sigma}$ measured along the MDC $A$ (MDC $B$ ) cuts $250 \mathrm{meV}$ above (below) the TP, as denoted in Fig. 4(a). (b),(c) Corresponding SARPES intensities and 3D spin polarization fitting for $\mathrm{TP}_{-}^{\sigma}$ and $\mathrm{TP}_{+}^{\sigma}$, respectively. Red and blue curves indicate the $B_{1,2,3,4}$ peak fitting, and surface-derived bands are in gray. (d) Analogous spin textures measured by rotating the sample by $180^{\circ}$; the frames in green and magenta indicate two inequivalent directions denoted in Fig. 1(a). (e) BSF constant energy maps; the black arrow indicates the Weyl-II fermion, and the dashed line its energy dispersion and hybridization with TP bands. (f) Close-ups of spin-resolved CEMs at -1.55 and $-1.6 \mathrm{eV}$, respectively, showing a change in spin winding number from \pm 1 to \pm 2 around the WP II point inclusion with a conspicuous "spin swirl" denoted by the dashed circle. 
system. Moreover, $\alpha$-GeTe is currently unique in the possibility to manipulate topological properties by temperature variation [24], ferroelectric switching [43], and/or magnetic doping [34].

M.F. acknowledges support from the Swiss National Science Foundation (SNSF) Project No. P2ELP2_181877 and G. S. from Project No. P30960-N27 of the Austrian Science Fund (FWF). J. M. and L. N. thank the CEDAMNF Project financed by the Ministry of Education, Youth and Sports of Czech Republic, Project No. CZ.02.1.01/0.0/0.0/ 15.003/0000358 and the Czech Science Foundation (GACR), Project No. 2018725S. M. G. acknowledges VEGA 1/0105/ 20 and Internal Research Grant System VVGS-2019-1227, V. V.V. acknowledges the Foundation for Polish Science through the IRA Program cofinanced by EU within SG OP, and finally O.C. acknowledges the financial support by the European Regional Development Fund Project CEITEC Nano+ (No. CZ.02.1.01/0.0/0.0/16_013/0001728). We thank Andrei Varykhalov at BESSY II for his support.

* Corresponding author. martin.gmitra@upjs.sk Corresponding author. juraj.krempasky@psi.ch

[1] C. L. Kane and E. J. Mele, Phys. Rev. Lett. 95, 226801 (2005).

[2] M. Z. Hasan and C. L. Kane, Rev. Mod. Phys. 82, 3045 (2010).

[3] D. Hsieh, Y. Xia, L. Wray, D. Qian, A. Pal, J. H. Dil, J. Osterwalder, F. Meier, G. Bihlmayer, C. L. Kane, Y. S. Hor, R. J. Cava, and M. Z. Hasan, Science 323, 919 (2009).

[4] A. Nishide, A. A. Taskin, Y. Takeichi, T. Okuda, A. Kakizaki, T. Hirahara, K. Nakatsuji, F. Komori, Y. Ando, and I. Matsuda, Phys. Rev. B 81, 041309(R) (2010).

[5] D. Hsieh, Y. Xia, D. Qian, L. Wray, J. H. Dil, F. Meier, J. Osterwalder, L. Patthey, J. G. Checkelsky, N. P. Ong, A. V. Fedorov, H. Lin, A. Bansil, D. Grauer, Y. S. Hor, R. J. Cava, and M.Z. Hasan, Nature (London) 460, 1101 (2009).

[6] Z.-H. Pan, E. Vescovo, A. V. Fedorov, D. Gardner, Y. S. Lee, S. Chu, G. D. Gu, and T. Valla, Phys. Rev. Lett. 106, 257004 (2011).

[7] K. Miyamoto, A. Kimura, T. Okuda, H. Miyahara, K. Kuroda, H. Namatame, M. Taniguchi, S. V. Eremeev, T. V. Menshchikova, E. V. Chulkov, K. A. Kokh, and O. E. Tereshchenko, Phys. Rev. Lett. 109, 166802 (2012).

[8] G. Landolt, S. Schreyeck, S. V. Eremeev, B. Slomski, S. Muff, J. Osterwalder, E. V. Chulkov, C. Gould, G. Karczewski, K. Brunner, H. Buhmann, L. W. Molenkamp, and J. H. Dil, Phys. Rev. Lett. 112, 057601 (2014).

[9] M. Z. Hasan and C. L. Kane, Rev. Mod. Phys. 82, 3045 (2010).

[10] B. Bradlyn, J. Cano, Z. Wang, M. G. Vergniory, C. Felser, R. J. Cava, and B.A. Bernevig, Science 353, aaf5037 (2016).

[11] B. Q. Lv, H. M. Weng, B. B. Fu, X. P. Wang, H. Miao, J. Ma, P. Richard, X. C. Huang, L. X. Zhao, G. F. Chen, Z.
Fang, X. Dai, T. Qian, and H. Ding, Phys. Rev. X 5, 031013 (2015).

[12] A. A. Soluyanov, D. Gresch, Z. Wang, Q. Wu, M. Troyer, X. Dai, and B. A. Bernevig, Nature (London) 527, 495 (2015).

[13] N. P. Armitage, E. J. Mele, and A. Vishwanath, Rev. Mod. Phys. 90, 015001 (2018).

[14] S.-Y. Xu et al., Science 349, 613 (2015).

[15] N. Hao and J. Hu, Natl. Sci. Rev. 6, 213 (2019).

[16] C. Fang, H. Weng, X. Dai, and Z. Fang, Chin. Phys. B 25, 117106 (2016).

[17] B. Q. Lv, Z.-L. Feng, Q.-N. Xu, X. Gao, J.-Z. Ma, L.-Y. Kong, P. Richard, Y.-B. Huang, V. N. Strocov, C. Fang, H.-M. Weng, Y.-G. Shi, T. Qian, and H. Ding, Nature (London) 546, 627 (2017).

[18] J.-Z. Ma et al., Nat. Phys. 14, 349 (2018).

[19] G. W. Winkler, Q. S. Wu, M. Troyer, P. Krogstrup, and A. A. Soluyanov, Phys. Rev. Lett. 117, 076403 (2016).

[20] S. Zaheer, S. M. Young, D. Cellucci, J. C. Y. Teo, C. L. Kane, E. J. Mele, and A. M. Rappe, Phys. Rev. B 87, 045202 (2013).

[21] G. W. Winkler, S. Singh, and A. A. Soluyanov, Chin. Phys. B 28, 077303 (2019).

[22] Z. Zhu, G. W. Winkler, Q. Wu, J. Li, and A. A. Soluyanov, Phys. Rev. X 6, 031003 (2016).

[23] D. Di Sante, P. Barone, R. Bertacco, and S. Picozzi, Adv. Mater. 25, 509 (2013).

[24] J. Krempaský, H. Volfová, S. Muff, N. Pilet, G. Landolt, M. Radović, M. Shi, D. Kriegner, V. Holý, J. Braun, H. Ebert, F. Bisti, V. A. Rogalev, V. N. Strocov, G. Springholz, J. Minár, and J. H. Dil, Phys. Rev. B 94, 205111 (2016).

[25] J. Krempaský, M. Fanciulli, N. Pilet, J. Minár, W. Khan, M. Muntwiler, F. Bertran, S. Muff, A. Weber, V. Strocov, V. Volobuiev, G. Springholz, and J. Dil, J. Phys. Chem. Solids 128, 237 (2017).

[26] D. Kriegner, G. Springholz, C. Richter, N. Pilet, E. Muller, M. Capron, H. Berger, V. Holý, J. H. Dil, and J. Krempaský, Crystals 9, 335 (2019).

[27] M. Hoesch, T. Greber, V. N. Petrov, M. Muntwiler, M. Hengsberger, W. Auwärter, and J. Osterwalder, J. Electron Spectrosc. Relat. Phenom. 124, 263 (2002).

[28] H. Ebert, D. Ködderitzsch, and J. Minár, Rep. Prog. Phys. 74 (2011).

[29] Ebert et al., The Munich SPR-KKR Package,Version 7.7, http://ebert.cup.uni-muenchen.de/SPRKKR.

[30] D. Takane, Z. Wang, S. Souma, K. Nakayama, T. Nakamura, H. Oinuma, Y. Nakata, H. Iwasawa, C. Cacho, T. Kim, K. Horiba, H. Kumigashira, T. Takahashi, Y. Ando, and T. Sato, Phys. Rev. Lett. 122, 076402 (2019).

[31] D. S. Sanchez et al., Nature (London) 567, 500 (2019).

[32] Z. Rao et al., Nature (London) 567, 496 (2019).

[33] See Supplemental Material at http://link.aps.org/supplemental/ 10.1103/PhysRevLett.126.206403 for animated band structure near TP, photon energy to momentum conversion, and additional SARPES data.

[34] J. Krempaský, S. Muff, F. Bisti, M. Fanciulli, H. Volfová, A. P. Weber, N. Pilet, P. Warnicke, F. Bertran, H. Ebert, J. Braun, J. Minár, G. Springholz, J. Dil, and V. Strocov, Nat. Commun. 7, 13071 (2016).

[35] G. Kremer, T. Jaouen, B. Salzmann, L. Nicolaï, M. Rumo, C. W. Nicholson, B. Hildebrand, J. H. Dil, J. Minár, G. 
Springholz, J. Krempaský, and C. Monney, Phys. Rev. Research 2, 033115 (2020).

[36] J. Krempaský, M. Fanciulli, L. Nicolaï, J. Minár, H. Volfová, O. Caha, V. V. Volobuev, J. Sánchez-Barriga, M. Gmitra, K. Yaji, K. Kuroda, S. Shin, F. Komori, G. Springholz, and J. H. Dil, Phys. Rev. Research 2, 013107 (2020).

[37] P. Zhang, P. Richard, T. Qian, Y.-M. Xu, X. Dai, and H. Ding, Rev. Sci. Instrum. 82, 043712 (2011).

[38] F. Meier, J. H. Dil, and J. Osterwalder, New J. Phys. 11, 125008 (2009).

[39] C.-H. Park and S. G. Louie, Phys. Rev. Lett. 109, 097601 (2012).
[40] J. Sánchez-Barriga, A. Varykhalov, J. Braun, S.-Y. Xu, N. Alidoust, O. Kornilov, J. Minár, K. Hummer, G. Springholz, G. Bauer, R. Schumann, L. V. Yashina, H. Ebert, M. Z. Hasan, and O. Rader, Phys. Rev. X 4, 011046 (2014).

[41] J. Henk, K. Miyamoto, and M. Donath, Phys. Rev. B 98, 045124 (2018).

[42] J. H. Dil, Electronic structure and magnetism of inorganic compounds 1, 023001 (2019).

[43] J. Krempaský, S. Muff, J. Minár, N. Pilet, M. Fanciulli, A. P. Weber, E. B. Guedes, M. Caputo, E. Müller, V. V. Volobuev, M. Gmitra, C. A. F. Vaz, V. Scagnoli, G. Springholz, and J. H. Dil, Phys. Rev. X 8, 021067 (2018). 